\title{
Enhancing effects on vacuole-targeting fungicidal activity of amphotericin B
}

\author{
Akira Ogita ${ }^{1,2}$, Ken-Ichi Fujita ${ }^{2}$ and Toshio Tanaka ${ }^{2}{ }^{*}$ \\ ${ }^{1}$ Research Center for Urban Health and Sports, Osaka City University, Osaka, Japan \\ 2 Department of Biology and Geosciences, Graduate School of Science, Osaka City University, Osaka, Japan
}

\section{Edited by:}

Bruce C. Campbell, Western Regional Research Centre, USA

\section{Reviewed by:}

Long Nam Nguyen, Singapore Immunological Network, Singapore

Nikhat Manzoor, Jamia Millia Islamia, India

\section{*Correspondence:}

Toshio Tanaka, Department of Biology and Geosciences, Graduate School of Science, Osaka City University,

3-3-138 Sugimoto, Sumiyoshi-ku, Osaka, Osaka, Japan.

e-mail: tanakato@sci.osaka-cu.ac.jp
Invasive fungal infections are major threats for immunocompromised patients as well as for those undergoing cancer chemotherapy. Amphotericin B (AmB), a classical antifungal drug with a polyene macrolide structure, is widely used for the control of serious fungal infections. However, the clinical use of this antifungal drug is limited by its side effects and the emergence of drug-resistant strains. AmB lethality has been generally attributed to alterations in plasma membrane ion permeability due to its specific binding to plasma membrane ergosterol. Recent studies with Saccharomyces cerevisiae and Candida albicans reveal the vacuole disruptive action as another cause of AmB lethality on the basis of marked amplification of its activity in combination with allicin, an allyl-sulfur compound from garlic. The enhancing effect of allicin is dependent on the inhibition of ergosteroltrafficking from the plasma membrane to the vacuole membrane, which is considered to be a cellular response to protect against disintegration of the vacuole membrane. The polyol macrolide niphimycin (NM) also possesses vacuole-targeting fungicidal activity, which is greater than that of AmB and nystatin. The alkyl side chain attached to the macrolide ring of NM is considered to possess an allicin-like inhibitory effect on the intracellular trafficking of ergosterol. The vacuole-targeting fungicidal activity was additionally detected with a bactericidal cyclic peptide polymyxin $\mathrm{B}(\mathrm{PMB})$, and was markedly enhanced when administered together with allicin, monensin, or salinomycin. The synergistic fungicidal activities of AmB and allicin may have significant implications for the development of vacuole-targeting chemotherapy against fungal infections.

Keywords: vacuole, fungicidal activity, amphotericin B, allicin

\section{INTRODUCTION}

The polyene macrolide antibiotic amphotericin B (AmB; Figure 1A) was introduced in the mid-1950s as the first effective antifungal drug for treating systemic mycoses (Johnson and Einstein, 2007). AmB has been used for controlling life-threatening systemic fungal infections primarily caused by Candida albicans and Aspergillus fumigatus (Lemke et al., 2005). The susceptibility of fungal cells to $\mathrm{AmB}$ absolutely requires the presence of ergosterol in the fungal plasma membrane. Molecular modeling studies indicate that $\mathrm{AmB}$ creates aqueous pores consisting of an annulus of eight molecules linked hydrophobically to ergosterol embedded in phospholipid bilayers (Baginski et al., 2006). This type of ion channel structure results in altered plasma membrane permeability and leakage of vital cytoplasmic components, such as $\mathrm{K}^{+}$, ultimately inducing cell death (Kerridge, 1980, 1985). However, AmB-induced lethal effects cannot be simply explained by the disturbance in plasma membrane ion transport function, because the leakage of $\mathrm{K}^{+}$has various secondary effects. Alonso et al. (1979) reported that AmB is a powerful inhibitor of both protein and ribonucleic acid synthesis in yeast cells at concentrations that do not affect the transport of amino acids or nucleosides. The induction of oxidative stress is also considered as an AmB-induced toxic event in C. albicans cells (Sokol-Anderson et al., 1986). The involvement of additional cytotoxic effects in AmB lethality is supported by the fact that the leakage of $\mathrm{K}^{+}$is not necessarily accompanied by the loss of cell viability (Chen et al., 1978). Clearly, the exact mechanism of AmB lethality is very complex and has not been completely deciphered.

Recently, AmB-induced cytoplasmic events have been visualized as vacuole membrane fragmentation into numerous dotted particles in Saccharomyces cerevisiae and C. albicans, and such drastic structural damage has been considered as the major cause of AmB lethality (Ogita et al., 2006; Borjihan et al., 2009). Vacuole membrane fragmentation is observed concurrently with AmB-induced cell death when this antibiotic is used at a nonlethal concentration in combination with allicin (Figure 1B), an allyl-sulfur compound derived from garlic (Ogita et al., 2006). This Mini Review describes a possible mechanism underlying the vacuole-targeting fungicidal activity of $\mathrm{AmB}$ and its enhancement by allicin. This review also deals with chemosensitization of the vacuole-targeting fungicidal activity detected with a compound other than AmB.

\section{ALLICIN AS AN ENHANCER OF THE FUNGICIDAL ACTIVITY OF AmB \\ Garlic (Allium sativum L.) has attracted much attention through- out human history as a medicinal panacea (Hobbs, 1992). A}




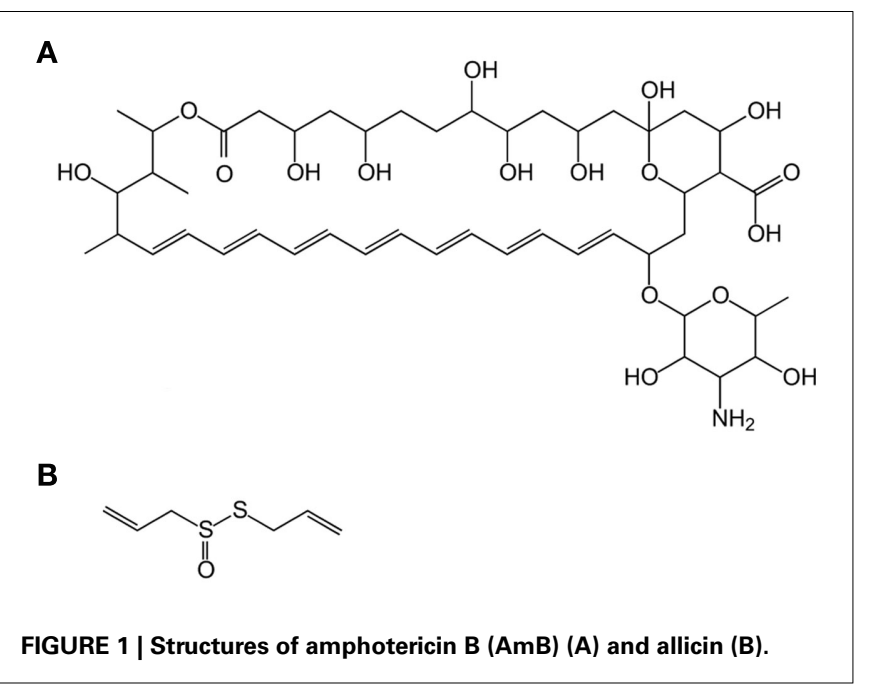

number of historical records attest to the protective effects of garlic preparations against a wide variety of microorganisms including bacteria, fungi, protozoa, and even viruses (Ankri and Mirelman, 1999). Garlic extract exerts additional pharmacological effects such as reducing serum neutral lipids and suppressing the development of mammary cancers in animals and the growth of human breast cancer cells in vitro (Jain et al., 1993; Tsubura et al., 2011).

Allicin (diallyl thiosulfinate) is the main active component in freshly crushed garlic extract that is responsible for protection against the proliferation of pathogenic microbes and malignant tumor cells (Ankri and Mirelman, 1999; Oommen et al., 2004). Its inhibitory activities on microbial and tumor cell growth may be partly elucidated by the inhibitory effect on certain thiol-containing enzymes via strong SH-modifying properties, as reflected by the production of $S$-allylmercaptocysteine from L-cysteine (Ankri and Mirelman, 1999; Miron et al., 2000; Rabinkov et al., 2000). Allicin is produced by the action of alliinase on the non-protein amino acid alliin that is abundant in garlic cloves (Stoll and Seebeck, 1951). Our own studies suggest the possibility of applying alliinase from the bacterium Ensifer adhaerens for in situ generation of fungicidal activity using alliin as a stable precursor (Yutani et al., 2011a). In addition to its own antimicrobial activity, allicin synergistically inhibits the growth of Mycobacterium tuberculosis when used in combination with either streptomycin or chloramphenicol (Gupta and Viswanathan, 1955; Ankri and Mirelman, 1999).

Allicin when added alone, inhibits the growth of $S$. cerevisiae cells (Ogita et al., 2005); however, it greatly potentiates the fungicidal activity of AmB (Ogita et al., 2006). AmB promotes $\mathrm{K}^{+}$efflux during the process of cell death, but this ion efflux was not increased significantly in the presence of allicin (Ogita et al., 2010b). This means that AmB-induced $\mathrm{K}^{+}$efflux could contribute to the lethality of $\mathrm{AmB}$ at least in part, but such an intracellular ionic imbalance is not a major cause in allicin-mediated enhancement of lethal cytotoxicity.
Fungal vacuole function is in osmoregulation, ion homeostasis, and cell volume regulation (Thumm, 2000; Wickner, 2000). Various hydrolytic enzymes, including proteases and nucleases, are thought to accumulate in vacuoles; hence, the damage to these organelles is considered a critical step in inducing cell death (Obara et al., 2001). In S. cerevisiae, vacuoles normally exhibit a swollen spherical architecture in untreated cells and are similarly observed in cells treated with either $120 \mu \mathrm{M}$ allicin or a non-lethal concentration of AmB (0.5 MM; Ogita et al., 2006, 2007b, 2010b). Surprisingly, the organelles are visible as small discrete dots in the cytoplasm when cells are treated with a lethal concentration of $\mathrm{AmB}(5 \mu \mathrm{M})$, suggesting that vacuole membrane damage is the primary mechanism of AmB's lethality (Ogita et al., 2006, 2007b, 2010b). Consistent with these findings is the detection of significant levels of AmB molecules in the cytoplasmic and plasma membrane fractions (Ogita et al., 2010b). Similar observations have been reported in studies on C. albicans (Borjihan et al., 2009).

\section{RELATIONSHIP BETWEEN AmB-INDUCED PLASMA AND VACUOLE MEMBRANE DAMAGE}

Although some controversy surrounds the lethal action of AmB, we believe that most researchers will agree that this antibiotic exerts its initial action at the fungal plasma membrane. According to the widely accepted hypothesis regarding the role of plasma membrane ergosterol, this neutral lipid is indispensable for the initial action of AmB (Ghannoum and Rice, 1999; Lemke et al., 2005). This view is also supported by findings that $\mathrm{AmB}$ resistance results from lack of ergosterol biosynthesis (Ellis, 2002). Chemotherapeutic application of $A m B$ is indeed based on its higher affinity and activity toward fungal plasma membranes containing ergosterol rather than cholesterol (Baginski et al., 2005). Brajtburg et al. (1980) discovered that the simultaneous addition of $\mathrm{K}^{+}$ and $\mathrm{Mg}^{2+}$ fully protects against the fungicidal activity of AmB. This fact supports the idea that AmB lethality is primarily dependent on the leakage of intracellular cations through the plasma membrane.

In contrast, Chen et al. (1978) reported that the aqueous channels or pores formed by $\mathrm{AmB}$ are not essential for its lethality for C. albicans, suggesting that the drug induces cell death by another mechanism. We have reported that the extracellular addition of $\mathrm{K}^{+}$and $\mathrm{Mg}^{2+}$ could restrict cytotoxicity as well as vacuole membrane fragmentation in $S$. cerevisiae treated with a lethal concentration of AmB alone (Ogita et al., 2010b). This may also suggest that AmB-induced leakage of these intracellular ions weakens the vacuole membrane or interferes with a mechanism of maintaining its normal rounded architecture. However, the simultaneous addition of allicin could still enhance AmB-induced cell death and the associated vacuole disruption. Our results reveal that, under certain conditions, AmB can cause vacuole disruption and cell death even when cells are protected against intracellular ionic imbalance. Therefore, we conclude that allicin likely inhibits a mechanism required for maintaining vacuole membrane stability that cannot be protected by simply increasing the intracellular cation concentration. 


\section{INTRACELLULAR ERGOSTEROL-TRAFFICKING AS A POSSIBLE TARGET OF ALLICIN}

Yeast vacuoles normally undergo fission as well as fusion, and defects in vacuole fusion cause vacuole membrane fragmentation (Seeley et al., 2002). Ergosterol is required for proper fluidity as well as the relating function of plasma membrane (Zinser et al., 1991), and thus plays an important role in both endocytosis (Munn et al., 1999) and homotypic vacuole fusion (Kato and Wickner, 2001). This suggests the possibility that fungal cells can increase their vacuole membrane ergosterol content by provoking ergosterol-trafficking from the plasma membrane in response to the preliminary vacuole membrane damage such as that caused by the action of AmB at a non-lethal concentration (Ogita et al., 2009a). In support of such an urgent response, ergosterol-enriched S. cerevisiae cells are protected against the vacuole membrane damage induced by $\mathrm{AmB}$ alone at a lethal concentration as well as that caused by the combination of allicin and AmB at nonlethal concentrations (Ogita et al., 2009a). C. albicans responded identically to the lethal actions of $\mathrm{AmB}$ when the cells had been pretreated with exogenously added ergosterol (Borjihan et al., 2009).

Isolated vacuoles represent a much simpler system than intact cells for the study of vacuole membrane fission and fusion. They lack not only consecutive transport systems, but also the principal energy-producing systems, and most of the metabolic and biosynthetic systems of the cell (Boller et al., 1975). Although allicin alone had no effect on the morphology of the isolated vacuoles, AmB could disrupt their swollen spherical architectures regardless of whether allicin was added to the vacuole suspension
(Borjihan et al., 2009; Ogita et al., 2009a). The vacuoles isolated from ergosterol-enriched cells are apparently resistant to the disruptive action of $\mathrm{AmB}$ when compared to those from untreated cells. The effect of allicin on the cellular localization of ergosterol could be visualized by filipin III. Ergosterol was, indeed, detected in the intracellular compartment corresponding to the vacuole membrane in addition to the plasma membrane when cells were treated with $\mathrm{AmB}$ alone at a non-lethal concentration (Ogita et al., 2009a). Unlikely, ergosterol remained in the plasma membrane without mobilization into the cytoplasm when allicin was added to the medium (Ogita et al., 2009a). These results suggest that the intracellular transport of ergosterol represents a protective reaction against the vacuole disruptive activity of $\mathrm{AmB}$.

A model for the role of allicin in the vacuole-targeting fungicidal activity of $\mathrm{AmB}$ has been proposed based on these results (Figure 2). In response to the action of $\mathrm{AmB}$, fungal cells increase the vacuole membrane ergosterol content by ergosterol-trafficking from the plasma membrane. As a result, at non-lethal concentrations of $\mathrm{AmB}$, cells can maintain the large, spherical architecture of the organelle (Figure 2A). In the presence of allicin, however, AmB can cause vacuole membrane damage even at a non-lethal concentration, because the vacuole membrane cannot be suitably provided with ergosterol due to failure of its trafficking from the plasma membrane (Figure 2B). In contrast, in ergosterol-enriched cells, the vacuole membrane is protected against AmB-induced disruptive damage even if the intracellular ergosterol transport is inhibited by allicin (Figure 2C).
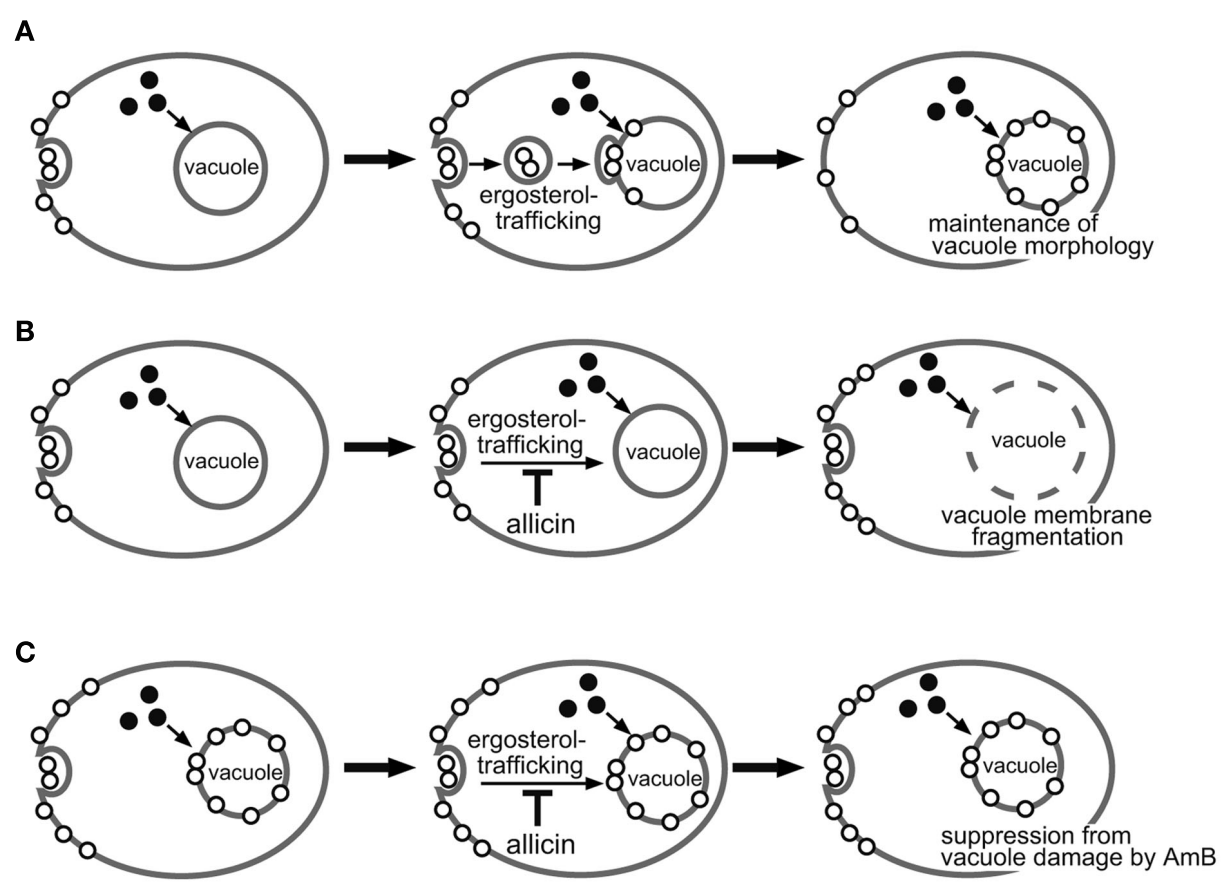

FIGURE 2 |A proposed model for the role of allicin and ergosterol $(O)$ in the vacuole-targeting fungicidal activity of amphotericin $B(A m B)(O)$. Untreated cells were incubated in the presence of $A m B$ alone at a non-lethal concentration (A) and AmB at a non-lethal concentration and allicin (B). Ergosterol-enriched cells were incubated in the presence of AmB at a non-lethal concentration and allicin (C). 


\section{FUNGICIDAL ACTIVITIES OF OTHER COMPOUNDS THAT TARGET VACUOLES}

Vacuole-targeting fungicidal activity has been markedly detected with the polyol macrolide antibiotic niphimycin (NM; Figure 3A) even in a nutrient medium (YPD) in which yeast cells are rather resistant to $\mathrm{AmB}$, possibly because of their high ergosterol content derived from "yeast extract," a component of YPD medium (Subbiah and Abplanalp, 2003; Ogita et al., 2010b). The molecular structure of NM is characterized by a polyol lactone ring and alkylguanidium chain attached to the ring, suggesting the contribution of the alkyl side chain to the predominant lethality of NM even in ergosterol-rich medium (Nakayama et al., 2002).

$N$-Methyl- $N^{\prime \prime}$-dodecylguanidine (MC12; Figure 3B) was synthesized and substituted for the alkyl side chain of NM, and was shown to strongly enhance the vacuole-targeting

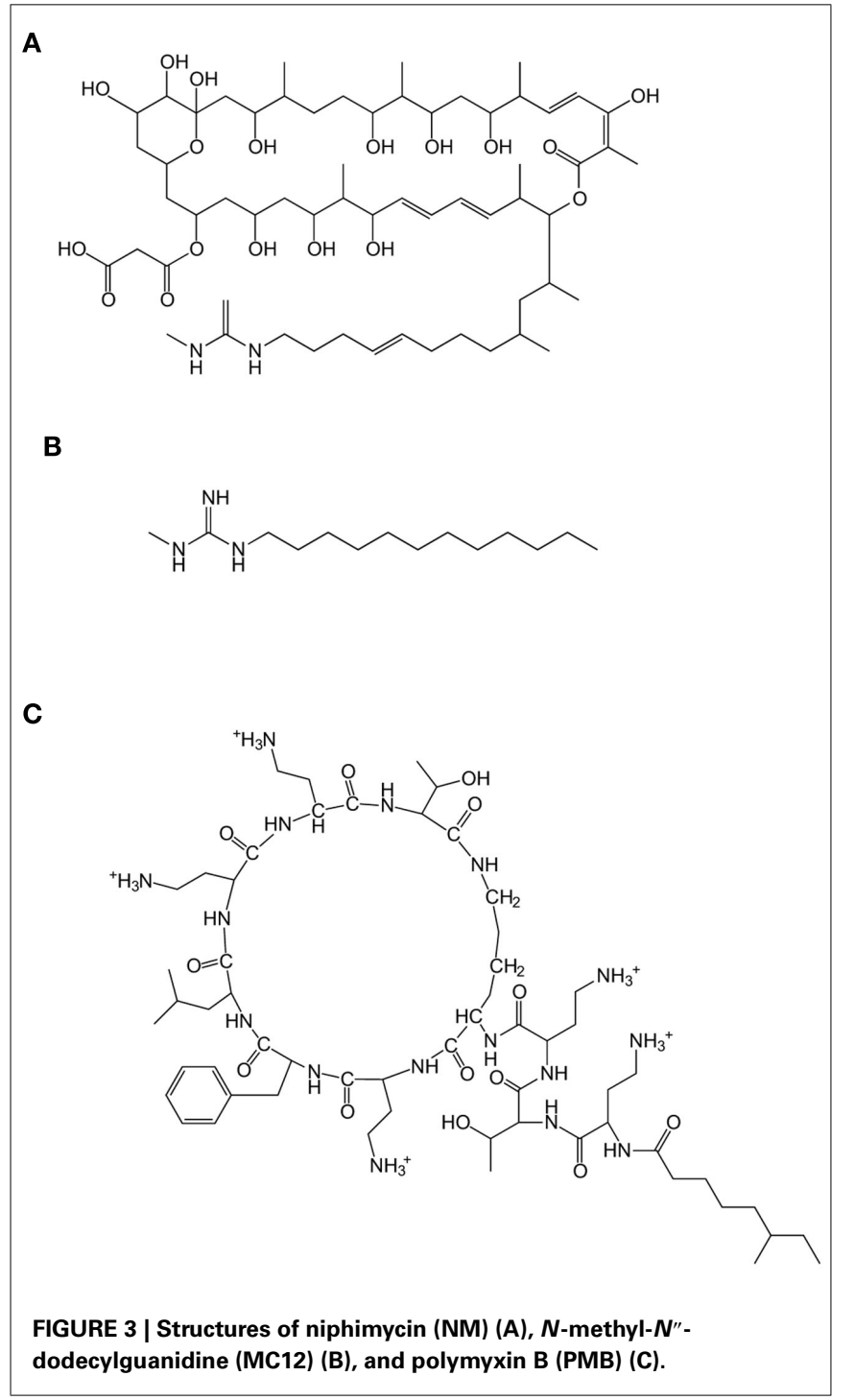

fungicidal activity of $\mathrm{AmB}$ against both $S$. cerevisiae and C. albicans (Usuki et al., 2000; Ogita et al., 2007a; Yutani et al., 2011b). The enhancing effect of MC12 on C. albicans pretreated with ergosterol was not observed, suggesting that it exerts an allicin-like inhibitory effect on intracellular ergosteroltrafficking.

The structure of the macrocyclic lactone ring of polyene macrolide compounds, in addition to the alkyl side chain, can also affect vacuole-targeting fungicidal activity. For example, nystatin possesses a polyene macrolide ring analogous to that of $\mathrm{AmB}$, and its fungicidal activity results in disruption of vacuoles as is the case for AmB. The fungicidal activity of nystatin is also enhanced in the presence of allicin in parallel to an enhancement of vacuole disruption (Ogita et al., 2010a). In contrast, allicin is ineffective in enhancing the fungicidal activity of filipin III, a pentaene macrolide that exhibits toxic effects such as inducing oxidative stress but does not disrupt vacuoles (Bonneau et al., 2010; Ogita et al., 2010a).

Polymyxin B (PMB; Figure 3C) is a bactericidal cyclic peptide effective against Gram-negative bacteria. $\mathrm{PMB}$ passes through the outer membrane, interacting with plasma membrane phospholipids, and thus, its bactericidal action is proposed to depend on a marked increase in plasma membrane permeability. This antibiotic can also interact with the plasma membranes of S. cerevisiae and C. albicans, but its fungicidal activity is much lower than that detected against bacterial cells. In our chemical screening experiment, $\mathrm{PMB}$ exhibited fungicidal activity that is enhanced by allicin (Ogita et al., 2007b). As expected, the vacuole membrane damage was clearly observed in PMB-treated S. cerevisiae and could be enhanced along with cytotoxicity even in ergosterol-rich medium.

The vacuole-targeting fungicidal activity of PMB against C. albicans, A. niger, and Mucor mucedo is enhanced in the presence of ionophores like monensin and salinomycin (Ogita et al., 2009b). An organosulfur compound, zwiebelane A, isolated from onion bulbs, also enhances the vacuole-targeting fungicidal activity of PMB (Borjihan et al., 2010). Interestingly, the enhancing effects of ionophores and zwiebelane A were observed when $S$. cerevisiae cells were treated with $\mathrm{PMB}$, but not with AmB, suggesting a difference in the mechanism of vacuole disruption between $\mathrm{AmB}$ and $\mathrm{PMB}$.

In spite of its proven track record, the well-known side effects of AmB might prevent its clinical use. Clinical use of AmB has been therefore improved with the aid of liposomal drug delivery systems that reduce its nephrotoxicity (Deray, 2002). A clinically effective dose of $\mathrm{AmB}$ may be also reduced when the antibiotic is used in combination with an agent like allicin. AmB resistance can be generated due to a genetic lack of plasma membrane ergosterol that plays an important role in the uptake of $\mathrm{AmB}$ into the fungal cytoplasm in addition to its participation in the formation of the plasma membrane's ion-permeable channel (Ogita et al., 2010b). Therefore, it seems possible to improve AmB-dependent chemotherapy by developing a method of enhancing its uptake into the cytoplasm of AmB-resistant fungal strains. 


\section{REFERENCES}

Alonso, M. A., Vázquez, D., and Carrasco, L. (1979). Compounds affecting membranes that inhibit protein synthesis in yeast. Antimicrob. Agents Chemother. 16, 750-756.

Ankri, S., and Mirelman, D. (1999). Antimicrobial properties of allicin from garlic. Microbes Infect. 1, 125-129.

Baginski, M., Czub, J., and Sternal, K. (2006). Interaction of amphotericin B and its selected derivatives with membranes: molecular modeling studies. Chem. Rec. 6, 320-332.

Baginski, M., Sternal, K., Czub, J., and Borowski, E. (2005). Molecular modelling of membrane activity of amphotericin B, a polyene macrolide antifungal antibiotic. Acta Biochim. Pol. 52, 655-658.

Boller, T., Dürr, M., and Wiemken, A. (1975). Characterization of a specific transport system for arginine in isolated yeast vacuoles. Eur. J. Biochem. 54, 81-91.

Bonneau, L., Gerbeau-Pissot, P., Thomas, D., Der, C., Lherminier, J., Bourque, S., Roche, Y., and SimonPlas, F. (2010). Plasma membrane sterol complexation, generated by filipin, triggers signaling responses in tobacco cells. Biochim. Biophys. Acta 1798, 2150-2159.

Borjihan, B., Ogita, A., Fujita, K., Doe, M., and Tanaka, T. (2010). The cyclic organosulfur compound zwiebelane A from onion (Allium cepa) functions as an enhancer of polymyxin B in fungal vacuole disruption. Planta Med. 76, 1864-1866.

Borjihan, H., Ogita, A., Fujita, K., Hirasawa, E., and Tanaka, T. (2009). The vacuole-targeting fungicidal activity of amphotericin B against the pathogenic fungus Candida albicans and its enhancement by allicin. J. Antibiot. 62, 691-697.

Brajtburg, J., Medoff, G., Kobayashi, G. S., and Elberg, S. (1980). Influence of extracellular $\mathrm{K}^{+}$or $\mathrm{Mg}^{2+}$ on the stages of the antifungal effects of amphotericin B and filipin. Antimicrob. Agents Chemother. 18, 593-597.

Chen, W. C., Chou, D. L., and Feingold, D. S. (1978). Dissociation between ion permeability and the lethal action of polyene antibiotics on Candida albicans. Antimicrob. Agents Chemother. 13, 914-917.

Deray, G. (2002). Amphotericin B nephrotoxicity. J. Antimicrob. Chemother. 49, 37-41.

Ellis, D. (2002). Amphotericin B: spectrum and resistance. J. Antimicrob. Chemother. 49, 7-10.

Ghannoum, M. A., and Rice, L. B. (1999). Antifungal agents: mode of action, mechanisms of resistance, and correlation of these mechanisms with bacterial resistance. Clin. Microbiol. Rev. 12, 501-517.

Gupta, K. C., and Viswanathan, R. (1955). Combined action of streptomycin and chloramphenicol with plant antibiotics against tubercle bacilli. I. Streptomycin and chloramphenicol with cepharanthine. II. Streptomycin and allicin. Antibiot. Chemother. 5, 24-27.

Hobbs, C. (1992). Garlic-the pungent panacea. Pharm. Hist. 34, 152-157.

Jain, A. K., Vargas, R., Gotzkowsky, S., and McMahon, F. G. (1993). Can garlic reduce levels of serum lipids? A controlled clinical study. Am. J. Med. 94, 632-635.

Johnson, R. H., and Einstein, H. E. (2007). Amphotericin B and coccidioidomycosis. Ann. N. Y. Acad. Sci. 1111, 434-441.

Kato, M., and Wickner, W. (2001). Ergosterol is required for the Sec18/ATP-dependent priming step of homotypic vacuole fusion. $E M B O$ J. 20, 4035-4040.

Kerridge, D. (1980). "The plasma membrane of Candida albicans and its role in the action of antifungal drugs," in The Eukaryotic Microbial Cell, eds G. W. Gooday, D. Lloyd, and A. P. J. Trinci (Cambridge: Cambridge University Press), 103.

Kerridge, D. (1985). "The protoplast membrane and antifungal drugs," in Fungal Protoplasts: Applications in Biochemistry and Genetics, eds J. F. Pebedy and L. Ferenczy (New York, NY: Marcel Dekker Inc.), 135.

Lemke, A., Kiderlen, A. F., and Kayser, O. (2005). Amphotericin B. Appl. Microbiol. Biotechnol. 68, 151-162.

Miron, T., Rabinkov, A., Mirelman, D., Wilchek, M., and Weiner, L. (2000). The mode of action of allicin: its ready permeability through phospholipid membranes may contribute to its biological activity. Biochim. Biophys. Acta 1463, 20-30.

Munn, A. L., Heese-Peck, A., Stevenson, B. J., Pichler, H., and Riezman, H. (1999). Specific sterols required for the internalization step of endocytosis in yeast. Mol. Biol. Cell 10, 3943-3957.

Nakayama, K., Yamaguchi, T., Doi, T., Usuki, Y., Taniguchi, M., and Tanaka, T. (2002). Synergistic combination of direct plasma membrane damage and oxidative stress as a cause of antifungal activity of polyol macrolide antibiotic niphimycin. $J$. Biosci. Bioeng. 94, 207-211.

Obara, K., Kuriyama, H., and Fukuda, H. (2001). Direct evidence of active and rapid nuclear degradation triggered by vacuole rupture during programmed cell death in Zinnia. Plant Physiol. 125, 615-626.

Ogita, A., Fujita, K., and Tanaka, T. (2009a). Enhancement of the fungicidal activity of amphotericin B by allicin: effects on intracellular ergosterol trafficking. Planta Med. 75, 222-226.

Ogita, A., Konishi, Y., Borjihan, B., Fujita, K., and Tanaka, T. (2009b). Synergistic fungicidal activities of polymyxin $\mathrm{B}$ and ionophores, and their dependence on direct disruptive action of polymyxin $B$ on fungal vacuole. J. Antibiot. 62, 81-87.

Ogita, A., Fujita, K., Taniguchi, M., and Tanaka, T. (2006). Enhancement of the fungicidal activity of amphotericin B by allicin, an allyl-sulfur compound from garlic, against the yeast Saccharomyces cerevisiae as a model system. Planta Med. 72, 1247-1250.

Ogita, A., Fujita, K., Usuki, Y., and Tanaka, T. (2010a). Targeted yeast vacuole disruption by polyene antibiotics with a macrocyclic lactone ring. Int. J. Antimicrob. Agents 35, 89-92.

Ogita, A., Yutani, M., Fujita, K., and Tanaka, T. (2010b). Dependence of vacuole disruption and independence of potassium ion efflux in fungicidal activity induced by combination of amphotericin B and allicin against Saccharomyces cerevisiae. J. Antibiot. 63, 689-692.

Ogita, A., Hirooka, K., Yamamoto, Y., Tsutsui, N., Fujita, K., Taniguchi, M., and Tanaka, T. (2005). Synergistic fungicidal activity of $\mathrm{Cu}^{2+}$ and allicin, an allyl sulfur compound from garlic, and its relation to the role of alkyl hydroperoxide reductase 1 as a cell surface defense in Saccharomyces cerevisiae. Toxicology 215, 205-213.

Ogita, A., Matsumoto, K., Fujita, K., Usuki, Y., Hatanaka, Y., and Tanaka, T. (2007a). Synergistic fungicidal activities of amphotericin $\mathrm{B}$ and $N$-methyl- $N^{\prime \prime}$-dodecylguanidine: a constituent of polyol macrolide antibiotic niphimycin. J. Antibiot. 60, 27-35.

Ogita, A., Nagao, Y., Fujita, K., and Tanaka, T. (2007b). Amplification of vacuole-targeting fungicidal activity of antibacterial antibiotic polymyxin $B$ by allicin, an allyl sulfur compound from garlic. J. Antibiot. 60, 511-518.

Oommen, S., Anto, R. J., Srinivas, G., and Karunagaran, D. (2004). Allicin (from garlic) induces caspasemediated apoptosis in cancer cells. Eur. J. Pharmacol. 485, 97-103.
Rabinkov, A., Miron, T., Mirelman, D., Wilchek, M., Glozman, S., Yavin, E., and Weiner, L. (2000). SAllylmercaptoglutathione: the reaction product of allicin with glutathione possesses SH-modifying and antioxidant properties. Biochim. Biophys. Acta 1499, 144-153.

Seeley, E. S., Kato, M., Margolis, N., Wickner, W., and Eitzen, G. (2002). Genomic analysis of homotypic vacuole fusion. Mol. Biol. Cell 13, 782-794.

Sokol-Anderson, M. L., Brajtburg, J., and Medoff, G. (1986). Amphotericin B-induced oxidative damage and killing of Candida albicans. J. Infect. Dis. 154, 76-83.

Stoll, A., and Seebeck, E. (1951). Chemical investigations on alliin, the specific principle of garlic. Adv. Enzymol. 11, 377-400.

Subbiah, M. T., and Abplanalp, W. (2003). Ergosterol (major sterol of baker's and brewer's yeast extracts) inhibits the growth of human breast cancer cells in vitro and the potential role of its oxidation products. Int. J. Vitam. Nutr. Res. 73, 19-23.

Thumm, M. (2000). Structure and function of the yeast vacuole and its role in autophagy. Microsc. Res. Tech. 51, 563-572.

Tsubura, A., Lai, Y. C., Kuwata, M., Uehara, N., and Yoshizawa, K. (2011). Anticancer effects of garlic and garlic-derived compounds for breast cancer control. Anticancer Agents Med. Chem. 11, 249-253.

Usuki, Y., Matsumoto, K., Inoue, T., Yoshioka, K., Iio, H., and Tanaka, T. (2000). Structure-activity relationship studies on niphimycin, a guanidylpolyol macrolide antibiotic. Part 1: the role of the $N$-methyl- $N^{\prime \prime}$ alkylguanidinium moiety. Bioorg. Med. Chem. Lett. 16, 1553-1556.

Wickner, W. (2000). Yeast vacuoles and membrane fusion pathways. EMBO J. 21, 1241-1247.

Yutani, M., Taniguchi, H., Borjihan, H., Ogita, A., Fujita, K., and Tanaka, T. (2011a). Alliinase from Ensifer adhaerens and its use for generation of fungicidal activity. $A M B$ Express 1, 2.

Yutani, M., Ogita, A., Usuki, Y., Fujita, K., and Tanaka, T. (2011b). Enhancement effect of $N$-methyl$N^{\prime \prime}$-dodecylguanidine on the vacuole-targeting fungicidal activity of amphotericin B against the pathogenic fungus Candida albicans. J. Antibiot. 64, 469-474.

Zinser, E., Sperka-Gottlieb, C. D., Fasch, E. V., Kohlwein, S. D., Paltauf, F., and Daum, G. (1991).Phospholipid 
synthesis and lipid composition of subcellular membranes in the unicellular eukaryote Saccharomyces cerevisiae. J. Bacteriol. 173, 2026-2034.

Conflict of Interest Statement: The authors declare that the research was conducted in the absence of any commercial or financial relationships that could be construed as a potential conflict of interest.

Received: 30 November 2011; accepted: 28 February 2012; published online: 19 March 2012.
Citation: Ogita A, Fujita K-I and Tanaka $T$ (2012) Enhancing effects on vacuoletargeting fungicidal activity of amphotericin B. Front. Microbio. 3:100. doi: 10.3389/fmicb.2012.00100

This article was submitted to Frontiers in Fungi and Their Interactions, a specialty of Frontiers in Microbiology.
Copyright (c) 2012 Ogita, Fujita and Tanaka. This is an open-access article distributed under the terms of the Creative Commons Attribution Non Commercial License, which permits non-commercial use, distribution, and reproduction in other forums, provided the original authors and source are credited. 